\title{
Localisation of collagen types and fibronectin in cartilage by immunofluorescence
}

\author{
HELEN B. EVANS, SHIRLEY AYAD, M. Z. ABEDIN, S. HOPKINS, \\ K. MORGAN, K. W. WALTON, * JACQUELINE B. WEISS, \\ AND P.J.L. HOLT
}

From the Departments of Rheumatology, University of Manchester Medical School, Manchester, and ${ }^{*}$ University of Birmingham Medical School, Birmingham

SUMmaRY Collagens type I, II, III, IV, and V and the minor cartilage collagens, $1 \alpha 2 \alpha 3 \alpha$, C-PS 1 , and C-PS 2, were purified, antibodies raised, and then used in immunofluorescence studies on bovine nasal cartilage (BNC). Punctate localisation was seen with the type II antibody. However, pretreatment of sections with hyaluronidase to remove the proteoglycans resulted in diffuse staining over all the section with this antibody. Antibodies to $1 \alpha 2 \alpha 3 \alpha$, C-PS 1 , and C-PS 2 collagens gave no staining on untreated BNC sections, but after treatment with hyaluronidase all 3 antibodies showed as a diffuse 'halo' round each chondrocyte lacuna. Anti-type I, anti-type III, and anti-type IV collagen antibodies did not stain untreated or enzyme treated BNC. Type V collagen antibodies gave a bright ring in the pericellular region of the lacunae of hyaluronidase-treated BNC. This was unexpected, as we could not detect type $V$ collagen biochemically in the same cartilage. Anti-fibronectin antibodies stained areas distant from the chondrocytes, these areas being distinct from those stained by $1 \alpha 2 \alpha 3 \alpha$ and C-PS antibodies, suggesting that fibronectin is not associated with these collagens in BNC. These results suggest that different collagen types may have different locations within the cartilage matrix, that proteoglycans may inhibit antibody association with collagen, and that fibronectin is normally not associated with all types of collagen.

At least 5 structurally and genetically distinct and well defined types of collagen have been identified. ${ }^{1}$ The predominant collagen of cartilage is type II, which has been localised in the cartilage matrix by specific antibodies. ${ }^{2}$ Proteoglycans of human and bovine hyaline cartilage have been purified and localised by immunofluorescent techniques. ${ }^{3}$

The presence in cartilage of small quantities of other collagenous components has been reported. Type $\mathrm{V}$ collagen, consisting of $\alpha 1$ (V) (previously called $\alpha \mathrm{B}$ ) chains only, has been described in epiphyseal cartilage ${ }^{4}$ and shown by immunofluorescence to be located pericellularly around the chondrocyte. ${ }^{5}$ Burgeson et al. ${ }^{6}$ isolated and partially characterised $3 \alpha$-sized collagen chains, designated $1 \alpha, 2 \alpha, 3 \alpha$, from human hyaline cartilage. The $1 \alpha$ and $2 \alpha$ chains are similar to the $\alpha$ chains of type $\mathrm{V}$ collagen but are genetically distinct. The $3 \alpha$ chain, although virtually identical with the $\alpha 1$ (II) chains of

Accepted for publication 8 September 1982.

Correspondence to Dr H. B. Evans, Department of Rheumatology, University of Manchester Medical School, Stopford Building, Oxford Road, Manchester M13 9PT. type II collagen, is more glycosylated and consistently copurifies with the other 2 chains. The collagens of bovine nasal cartilage (BNC) have been studied by Ayad et al.,${ }^{78}$ whoidentified type II and $1 \alpha$ $2 \alpha 3 \alpha$ collagens and 2 new collagens designated C-PS 1 and C-PS 2. C-PS 1 and C-PS 2 collagens are short triple helical collagens approximately one-third and one-seventh the length of type II collagen respectively and the constituent chains are linked by interchain disulphide bonds.

This report describes the localisation of these collagens in BNC by immunofluorescence.

\section{Materials and methods}

Collagens. These were isolated from various bovine tissues after limited pepsin digestion. Type II, $1 \alpha 2 \alpha$ $3 \alpha$, C-PS 1 , and C-PS 2 collagens were isolated from adult nasal cartilage as described by Ayad et al. ${ }^{78}$ Types I and III collagen were prepared from placental membranes according to Epstein. ${ }^{9}$ Types IV and $\mathrm{V}$ collagen were isolated from placental villi and membrane respectively by the method of Abedin et 
$a l .{ }^{10}$ Each collagen was purified by repeated precipitation according to its specific solubility properties in $0.02 \mathrm{M} \mathrm{Na}_{2} \mathrm{HPO}_{4}$ (pH 9.2), phosphate-buffered saline (PBS) (pH 7.2) and 0.5-5.0 M NaCl. 0.05 M tris $\mathrm{HC} 1(\mathrm{pH} \mathrm{7 \cdot 4)}$. The purity of these preparations was checked by sodium dodecyl sulphate polyacrylamide gel electrophoresis.

Antisera to collagen. Antisera to bovine native type II collagen were raised in rats injected intradermally with an emulsion of type II collagen in incomplete Freund's adjuvant (ICFA, Miles), by the regimen of Morgan et al. ${ }^{11}$ A proportion of rats developed type II collagen-induced arthritis, ${ }^{12}$ and separate pools of sera from arthritic and nonarthritic rats were made. IgG antibodies to native type II collagen in each pool were measured by radioimmunoassay, ${ }^{11}$ and were $87 \cdot 1 \mu \mathrm{g}{ }^{125} \mathrm{I}$ labelled anti-rat $\mathrm{IgG}$ bound/ml of serum for the arthritic pool and 59.6 $\mu \mathrm{g}$ for the nonarthritic pool.

Antisera to bovine native types I-V, $1 \alpha 2 \alpha$ $3 \alpha$, C-PS 1 , and C-PS 2 collagens were raised in guinea-pigs by an immunisation regimen similar to that of Beard et al. ${ }^{13}$ Guinea-pigs were inoculated intradermally with $500 \mu \mathrm{g}$ of collagen in complete Freund's adjuvant (Difco) and boosted intradermally 2-3 weeks later with $250 \mu \mathrm{g}$ collagen in ICFA (Difco). Two weeks after the boost animals were bled out by cardiac puncture under halothane anaesthesia.

Immunoabsorption of antisera. $1 \alpha 2 \alpha 3 \alpha$, C-PS 1 , C-PS 2, types I, II, III, IV, and V collagen were coupled to cyanogen-bromide-activated sepharose (Pharmacia), by a modification, described in the manufacturer's literature, of the method of Axen et al. ${ }^{14}$ Each antiserum was purified by absorption against the heterologous collagens and finally absorbed on to a column of the homologous collagen. Specific antibodies were eluted with glycine- $\mathrm{HCl}$ buffer, $0 \cdot 2$ $\mathrm{M}, \mathrm{pH} 2 \cdot 2$, neutralised, freeze-dried, and reconstituted in PBS at $1 \mathrm{mg} / \mathrm{ml}$. Antibodies were routinely diluted $1 / 10$ and $1 / 20$ before use.

Antiserum to fibronectin. Antiserum raised in rabbits against human fibronectin was obtained commercially (Miles Laboratories Ltd). The product was an IgG fraction of the antiserum and gave identical results to a fibronectin antiserum, also used, which was raised as described by Scott et al. ${ }^{15}$

Sectioning of BNC and enzyme treatments. Blocks of BNC were snap-frozen in n-isopentane cooled by liquid nitrogen and stored at $-70^{\circ} \mathrm{C}$. Sections $5 \mu \mathrm{m}$ thick were cut on a cryostat and transferred to microscope slides coated with a solution of $2 \%$ Bostik clear adhesive (Bostik Ltd, Leicester) in acetone. Pretreatment of slides in this way assisted adherence of sections. To expose collagen by removal of the proteoglycans, sections were treated with hyaluronidase (Worthington Biochemical Corporation, New
Jersey), diluted to 186 units $/ \mathrm{ml}$ in $0.025 \mathrm{M}$ $\mathrm{NaCl} / 0 \cdot 05 \mathrm{M}$ acetate buffer, $\mathrm{pH} 5 \cdot 0$. Sections were incubated with the enzyme for 30 minutes at room temperature, then washed in PBS before staining. Some other sections were incubated with bacterial collagenase R (Calbiochem, San Diego, California), at $38 \mathrm{units} / \mathrm{ml}$ for 30 minutes at room temperature and washed with PBS before use.

Alcian blue and Sirius red stains. Sections were stained for proteoglycan with alcian blue, $\mathrm{pH} 2 \cdot 5$, by the method of Lison ${ }^{16}$ and for collagen with Sirius red by the method of Sweat et al. $^{17}$

Immunofluorescence. Sections were incubated with collagen antisera for 30 minutes washed with 3 changes of PBS ( $30 \mathrm{~min})$ and then stained with either FITC-conjugated goat anti-guinea-pig immunoglobulins, FITC-conjugated rabbit anti-rat immunoglobulins, or FITC-conjugated swine anti-rabbit immunoglobulins (Nordic Immunological Laboratories Ltd, Maidenhead, Berks), as appropriate. After further washing in PBS, sections were mounted in glycerol/saline and observed with a Vickers M41 Photoplan microscope, fitted with a xenon lamp. Controls included substituting normal rat serum, normal guinea-pig serum, and PBS for anti-collagen antibodies in the first stage and blocking the fluorescent staining with unlabelled anti-rat or anti-guineapig immunoglobulins.

\section{Results}

DESCRIPTION OF CARTILAGE.

Regions of the cartilage matrix are described according to Poole et al. ${ }^{3}$ (Fig. 1). The zone at the surface of the chondrocyte and at the edge of the lacuna is referred to as the pericellular area. The wider zone surrounding this is the territorial zone, and the region between the territorial zones is the interterritorial matrix.

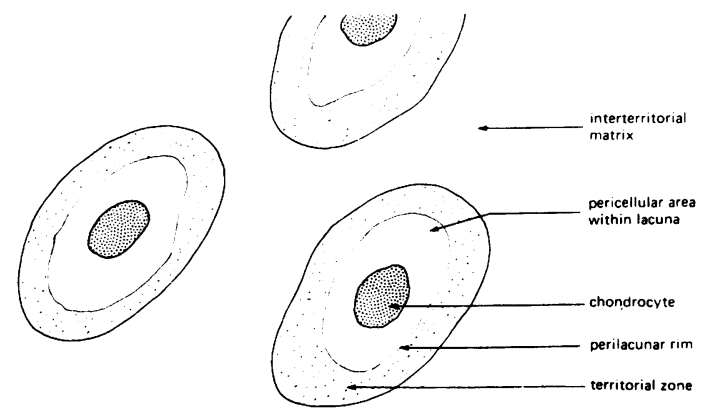

Fig. 1 Areas of the cartilage matrix. The zone at the surface of the chondrocyte is referred to as the pericellular area. The wider zone surrounding this is the territorial zone, and the region between territorial zones is the interterritorial matrix. 


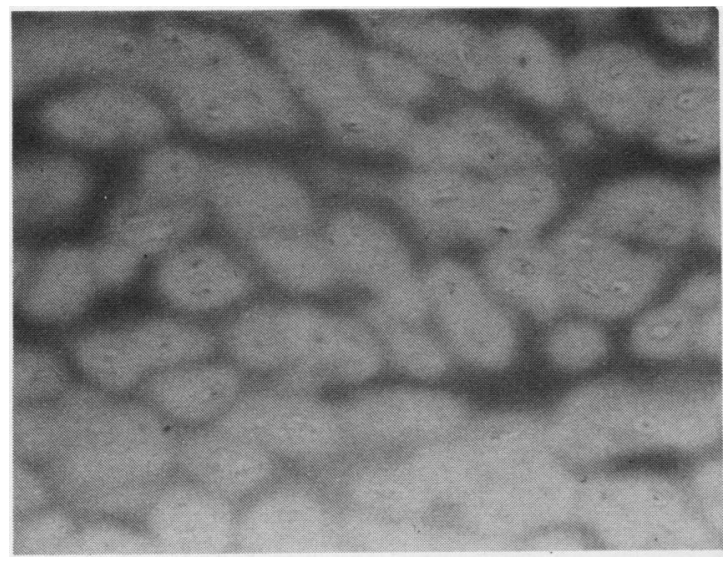

Fig. 2 BNC stained with Sirius red. $(\times 470)$.

HISTOCHEMICAL STAINING OF BNC

A section of untreated BNC stained with Sirius red is shown in Fig. 2. Fig. 3 shows a section of BNC treated with hyaluronidase and stained with Sirius red. Hyaluronidase treatment resulted in increased, more dense staining, especially in the territorial zone, indicating that more collagen was exposed. Figs 4 and 5 show BNC stained with alcian blue, untreated and after hyaluronidase treatment respectively. Decreased staining after treatment indicates that proteoglycan has been removed by the enzyme. Hyaluronidase treatment frequently resulted in displacement of chondrocytes, which were washed away during the staining procedures, as the sections were not fixed.

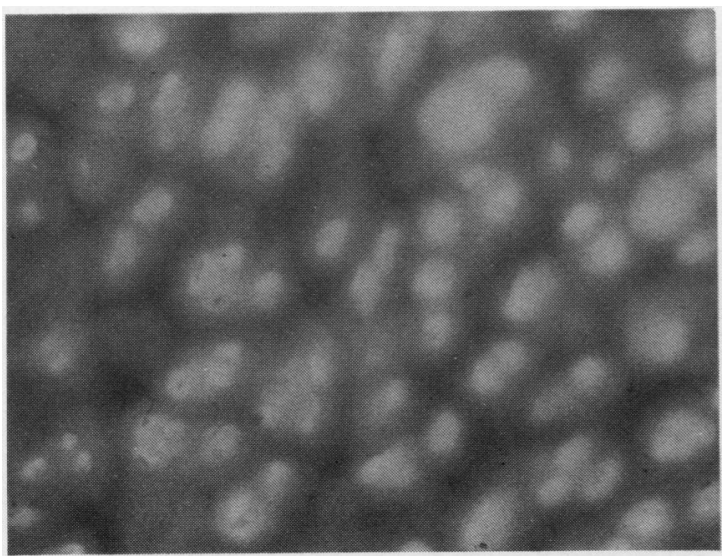

Fig. 3 BNC treated with hyaluronidase, then stained with Sirius red. $(\times 470)$.

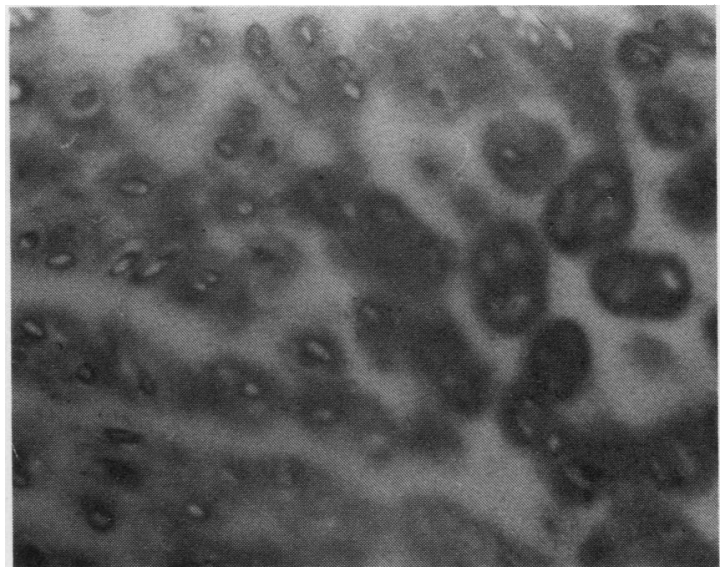

Fig. 4 BNC stained with alcian blue. $(\times 470)$.

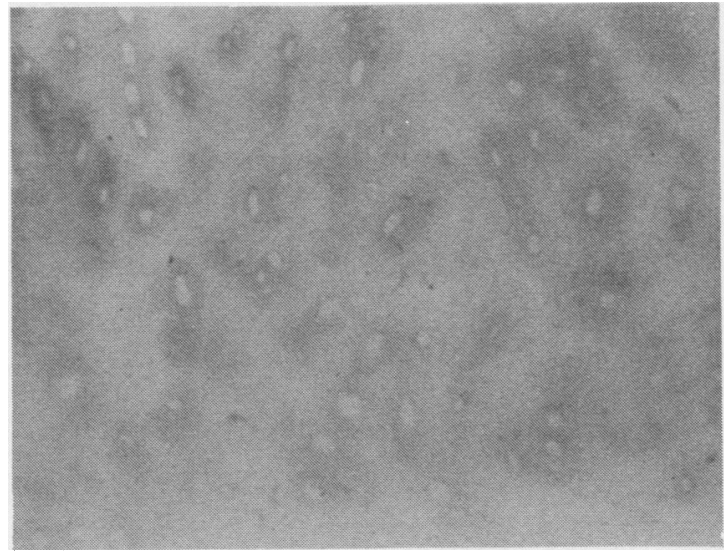

Fig. 5 BNC treated with hyaluronidase, then stained with alcian blue. $(\times 470)$.

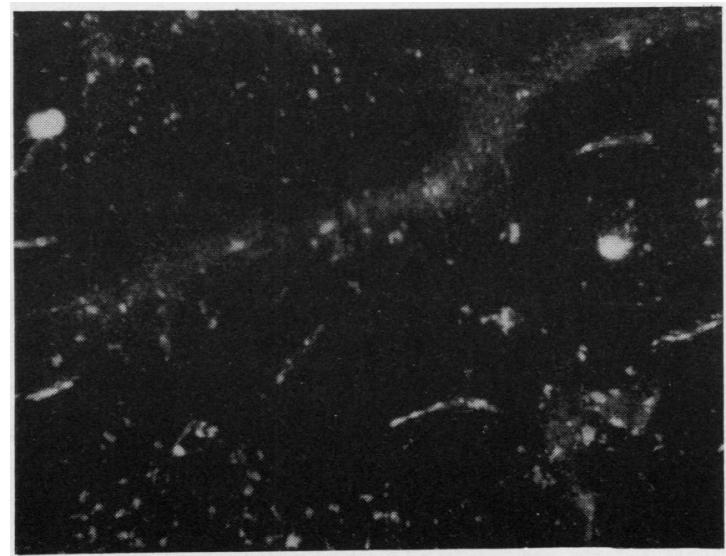

Fig. 6 Immunolocalisation of type II collagen on untreated BNC. $(\times 470)$. 


\section{IMMUNOFLUORESCENCE}

Type II collagen. Immunolocalisation of type II collagen on untreated BNC gave discrete groups of fluorescent spots in the matrix (Fig. 6). Chondrocytes stained brightly, but, as this was also seen in controls with normal rat serum and unconjugated blocking antibodies (Figs 7 and 8), it was considered to be nonspecific. Diffuse staining over the cartilage matrix was observed on hyaluronidase-treated sections (Fig.

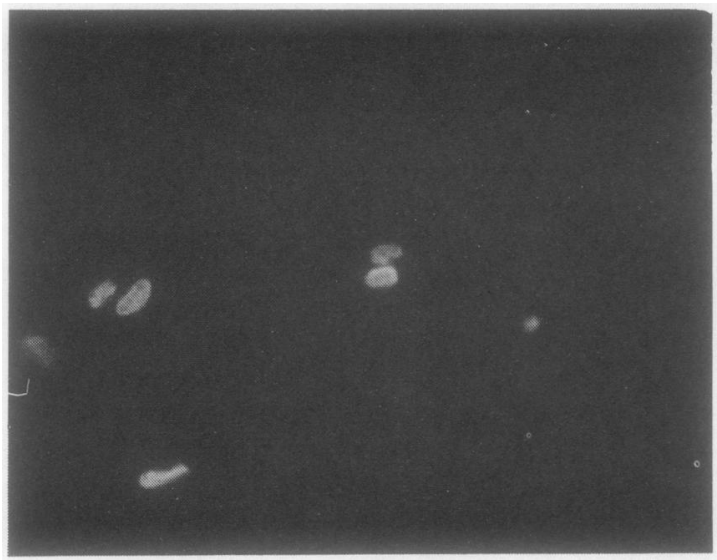

Fig. 7 Control. Untreated BNC section overlaid with normal rat serum (1/10 dilution), washed, treated with FITC-labelled rabbit anti-rat immunoglobulins, and washed again before mounting. $(\times 470)$.

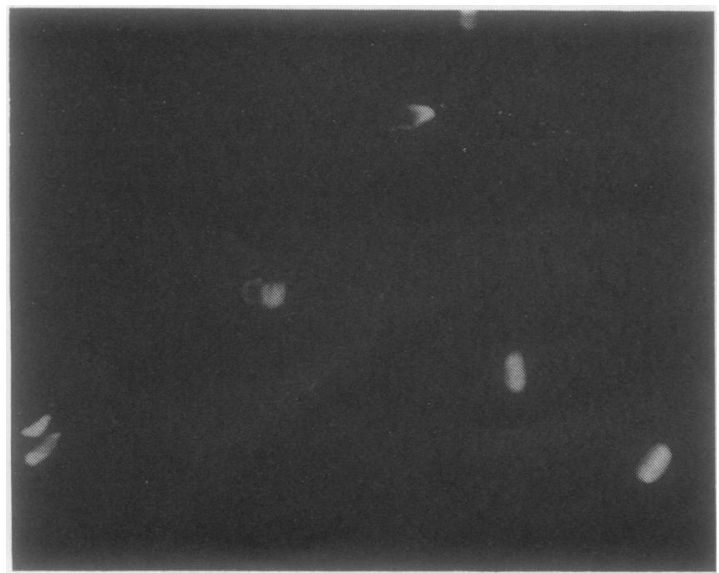

Fig. 8 Control. Untreated BNC section overlaid with rat anti-type II collagen, washed, overlaid with unconjugated rabbit anti-rat immunoglobulins (1/5 dilution), washed and overlaid with FITC-labelled rabbit anti-rat immunoglobulins, and washed again before mounting. $(\times 470)$.
9), which was absent in controls. Qualitatively similar staining patterns were observed with sera from arthritic and nonarthritic rats.

Staining with anti-type II collagen antisera was abolished by treatment of sections with bacterial collagenase (Fig. 10).

$1 \alpha 2 \alpha 3 \alpha$ collagen. Only nonspecific staining of chondrocytes was seen on untreated BNC sections stained with antibodies to $1 \alpha 2 \alpha 3 \alpha$ collagen. However, after treatment with hyaluronidase, diffuse staining of the territorial matrix was obtained (Fig. 11). This was considered to be specific, as the staining was abolished by blocking controls.

$C$-PS 1 and C-PS 2 collagens. The staining patterns for these 2 collagens were similar to that for $1 \alpha 2 \alpha 3 \alpha$

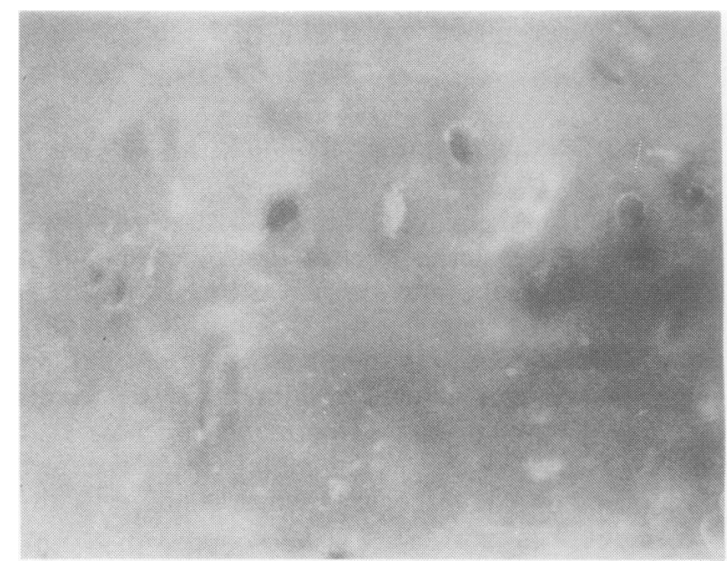

Fig. 9 BNC treated with hyaluronidase.

Immunolocalisation of type II collagen. $(\times 470)$.

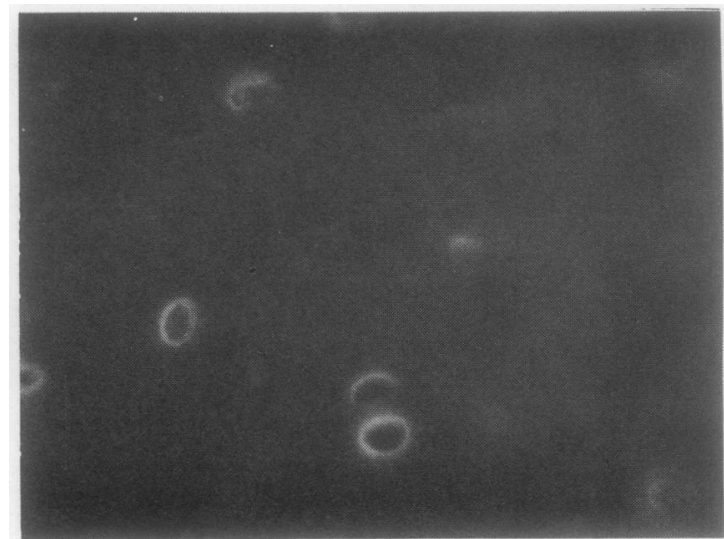

Fig. 10 BNC treated with bacterial collagenase, and stained for type II collagen. $(\times 470)$. 
collagen (Figs 12 and 13). Staining was abolished by blocking controls.

Type $V$ collagen. Again, only nonspecific staining of chondrocytes was seen on untreated BNC sections. On hyaluronidase-treated sections very bright, discrete pericellular staining was observed (Fig. 14). This was considered specific by the criteria of using normal guinea-pig serum and blocking controls.

Types I, III, and IV collagen. No staining was seen with antibodies to these 3 collagens, either on untreated BNC or on sections treated with hyaluronidase.

Fibronectin. Anti-fibronectin antibodies stained the interterritorial matrix on both untreated and hyaluronidase-treated sections (Fig. 15), and this was shown to be specific by controls with normal rabbit serum and blocking controls. No staining of the pericellular and territorial region was seen.

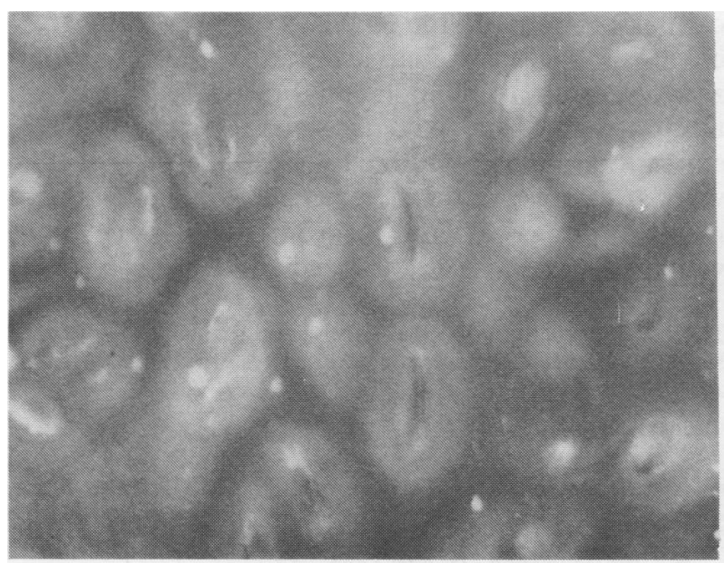

Fig. $11 B N C$ treated with hyaluronidase.

Immunolocalisation of $1 \alpha 2 \alpha 3 \alpha$ collagen. $(\times 470)$.

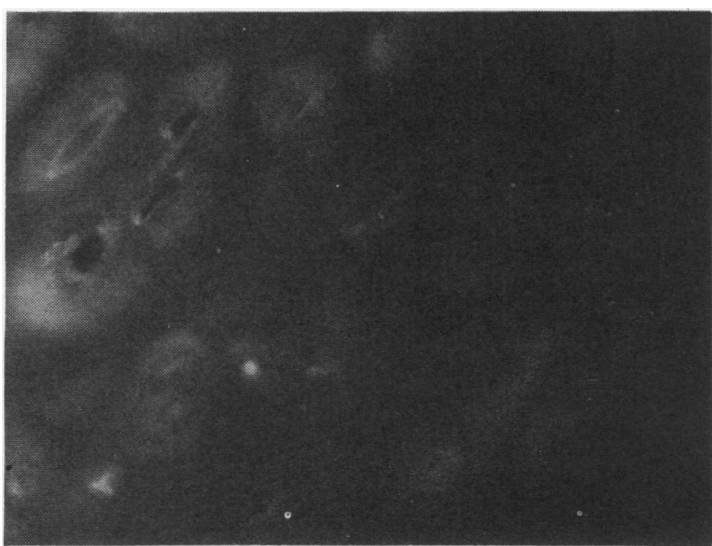

Fig. $12 B N C$ treated with hyaluronidase.

Immunolocalisation of C-PS 1 collagen. $(\times 470)$.

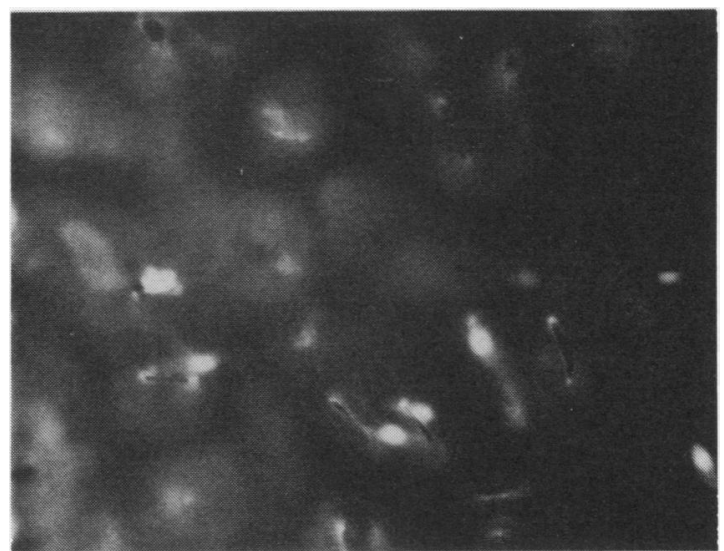

Fig. 13 BNC treated with hyaluronidase.

Immunolocalisation of C-PS 2 collagen. $(\times 470)$.

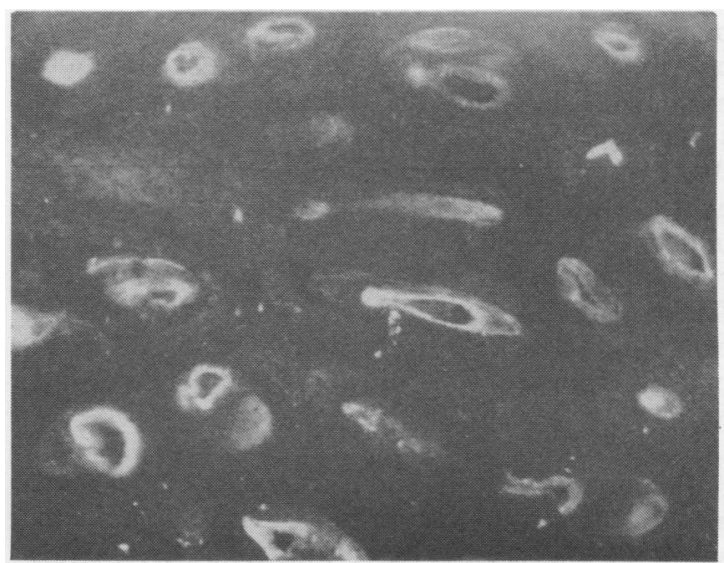

Fig. 14 BNC treated with hyaluronidase.

Immunolocalisation of type $V$ collagen. $(\times 470)$.

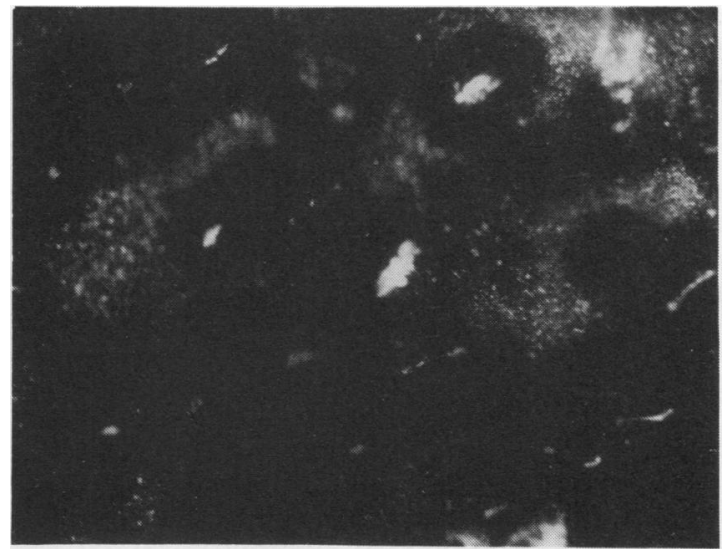

Fig. $15 B N C$, untreated. Immunolocalisation of fibronectin. $(\times 470)$. 


\section{Discussion}

This study suggests that different collagen types are distributed in defined areas within the cartilage matrix. Type II collagen, the most abundant collagen type in cartilage, appears on untreated sections to be distributed interterritorially. However, after treatment with hyaluronidase it can be seen that type II collagen is present throughout the cartilage matrix. In contrast, $1 \alpha 2 \alpha 3 \alpha$ collagen and the C-PS collagens cannot be detected on untreated sections of BNC and can only be visualised after enzyme treatment to remove proteoglycans. It would appear that these collagens are located around the chondrocytes, in the territorial zone, and that they are either absent, or present in undetectable quantities, in the interterritorial spaces.

Type $\mathrm{V}$ collagen presents an interesting anomaly. Although there is one report of type $V$ collagen being biochemically detected in cartilage, ${ }^{4}$ this finding has not been confirmed by us using biochemical methods. $^{7}$ The type $\mathrm{V}$ antiserum was extensively absorbed against other collagens and gave the expected distribution on placental and other tissues (Evans and Abedin, unpublished observations), so it seems unlikely that the poor specificity of this reagent was responsible for an artefactual result. Immunofluorescent studies are renowned for the production of 'edge' effects, where nonspecific staining appears on the edge of sections. It could be argued that such an effect was observed with the type $V$ antiserum, but this is unlikely, as the immunofluorescence was abolished by blocking controls. Two possibilities could explain the staining with the type $\mathrm{V}$ antiserum; the antibodies may cross-react with a hitherto undiscovered collagen present in cartilage which is present in low quantities or is intimately associated with other constituents of cartilage. Alternatively, type $\mathrm{V}$ collagen may indeed be present in cartilage, and its detection by immunohistochemical means but not by biochemical methods may reflect the differing sensitivities of the 2 techniques. It is interesting to note that the fluorescent patterns on cartilage which have been described with sera from patients suffering from relapsing polychondritis ${ }^{1819}$ are similar to that observed with our type $V$ antiserum. Previously these antibodies have been assumed to be directed against type II collagen.

Antibodies to fibronectin failed to stain frozen sections of the cartilage matrix of chick sternal cartilage. ${ }^{20}$ However, these authors showed that chondrocytes from this cartilage in culture synthesise and secrete fibronectin, indicating that these cells do have the potential to produce fibronectin under certain conditions. Our study suggests that fibronectin is present in adult bovine nasal cartilage matrix. It locates only in the interterritorial matrix, in the areas from which $1 \alpha 2 \alpha 3 \alpha$ and the C-PS collagens appear to be absent. The only type of collagen we have detected in this region is type II.

Poole et al. ${ }^{3}$ have shown in an immunohistochemical study of proteoglycans and link protein of bovine articular cartilage that these have well-defined locations. Proteoglycan was evenly distributed throughout the cartilage matrix, yet more link protein was detectable in the interterritorial sites in the middle and deep zones of the bovine articular cartilage. Well-defined zones of weaker territorial staining for link protein stained strongest for chondroitin sulphate.

Our study shows that collagen types have welldefined locations in bovine nasal cartilage. The functional significance of this is not yet clear. C-PS 1 and C-PS 2 collagens probably exist in a much larger form in the intact tissue. ${ }^{8}$ Moreover, they could be specifically associated with the $1 \alpha 2 \alpha 3 \alpha$ collagen, since all 3 collagens have a similar location in the territorial zone. Such an association could provide the protective enclosures believed by several workers ${ }^{21} 22$ to exist around the chondrocytes. An extension of this work at the level of the electron microscope might be more informative with regard to this suggestion, which at present is purely speculative.

The work was supported by the Arthritis and Rheumatism Council, Medical Research Council, and the North Western Regional Health Authority.

\section{References}

1 Bornstein P, Sage H. Structurally distinct collagen types. Ann Rev Biochem 1980; 49: 957-1003.

2 Von der Mark K, Von der Mark H, Gay S. Study of differential collagen synthesis during development of the chick embryo by immunofluorescence. II Localisation of type I and type II collagen during long bone development. Dev Biol 1976; 53: 153-70.

3 Poole A R, Pidoux I, Reiner A, Tang L-H, Choi H, Rosenburg L. Localisation of proteoglycan monomer and link protein in the matrix of bovine articular cartilage. J Histochem Cytochem 1980; 28: 621-35.

4 Rhodes R K, Miller E J. Physicochemical characterisation and molecular organisation of the collagen $A$ and $B$ chains. Biochemistry 1978; 17: 3342-8.

5 Gay S, Rhodes R K, Gay R E, Miller E J. Collagen molecules comprised of $\alpha 1(\mathrm{~V})$-chains (B-chains): an apparent localisation in the exocytoskeleton. Collagen Res 1981; 1: 53-8.

6 Burgeson R E, Hollister D W. Collagen heterogenieity in human cartilage: identification of several new collagen chains. Biochem Biophys Res Commun 1979; 87: 1124-31.

7 Ayad S, Abedin M Z, Grundy S M, Weiss J B. Isolation and characterisation of an unusual collagen from hyaline cartilage and intervertebral disc. FEBS Lett 1981; 123: 195-9.

8 Ayad S, Abedin M Z, Weiss J B, Grundy S M. Characterisation of another short-chain disulphide-bonded collagen from cartilage, vitreous and intervertebral disc. FEBS Lett 1982; 139: 300-4. 
9 Epstein E H. $[\alpha 1 \text { (III) }]_{3}$ human skin collagen. Release by pepsin digestion and preponderance in foetal life. $\mathrm{J} \mathrm{Biol} \mathrm{Chem}$ 1974; 249: 3225-31.

10 Abedin $M$ Z, Ayad S, Weiss J B. Type V collagen: the presence of appreciable amounts of $\alpha_{3}(\mathrm{~V})$ chain in uterus. Biochem Biophys Res Commun 1981; 102: 1237-45.

11 Morgan K, Clague R B, Shaw M J, Holt P J L. Native type II collagen-induced arthritis in the rat. I. Incidence and humoral response to collagen. Ann Rhuem Dis 1980; 39: 285-90.

12 Trentham D E, Townes A S, Kang A H. Autoimmunity to type II collagen: an experimental model of arthritis. $J$ Exp Med 1977; 146: 857-67.

13 Beard H K, Ueda M, Faulk W P, Glynn L E. Cell-mediated and humoral immunity to chick type II collagen and its cyanogen bromide peptides in guinea-pigs. Immunology 1978; 34: 323-35.

14 Axen R, Brath J, Ernback S. Chemical coupling of peptides and proteins to polysaccharides by means of cyanogen halides. Nature 1967; 214: 1302-4.

15 Scott D L, Bedford P A, Walton K W. The preparation of fibronectin antigen and antiserum. J Immunol Methods 1981; 43: 29-38.
16 Lison L. Alcian blue $8 \mathrm{G}$ with chlorantine fast red 5B. A technic for selective staining of mucopolysaccharides. Stain Technol 1954; 29: 131-8.

17 Sweat F, Puchtler H, Rosenthal S I. Sirius red F3BA as a stain for connective tissue. Arch Pathol 1964; 78: 69-72.

18 Dolan D L, Leramon G B, Teitelbaum S L. Relapsing polychondritis. Analytical literature review and studies on pathogenesis. Am J Med 1966; 41: 285-99.

19 Rogers P H, Boden G, Tourtellote C D. Relapsing polychondritis with insulin resistance and antibodies to cartilage. Am J Med 1973; 55: 243-8.

20 Dessau W, Sasse J, Timpl R, Jilck F, Von der Mark K. Synthesis and extracellular deposition of fibronectin in chondrocyte cultures. Response to the removal of extracellular cartilage matrix.J Cell Biol 1978; 79: 342-55.

21 Szirmai J A. Structure of cartilage. In: Engle A, Larsson T, eds. Ageing of cunnective and skeletal tissue. Stockholm: Nordiska Bokhadelns, 1969: 163-84.

22 Mulholland R. Lateral hydraulic permeability and morphology of articular cartilage. In: Ali S Y, Elves M W, Leaback D H, eds. Normal and osteoarthrotic articular cartilage. London: Institute of Orthopaedics, 1974: 85-101. 